\title{
Optimal Power Flow Using firefly-algorithm
}

\author{
K. Karboune *, I.Chaib ${ }^{1}$, S. Arif and A.choucha \\ Energy Systems and Electrical Grid Control Analysis Laboratory (LACOSERE). \\ University Amar Telidji of Laghouat, BP-37G-03000 Laghouat, Algeria. \\ *Corresponding author, e-mail: k.karboune@lagh-univ.dz.
}

Received: April 12, 2021

Accepted: May 25, 2021

\begin{abstract}
In this paper, the Firefly Algorithm (FA) is proposed to find the optimal solution for the Optimal Power Flow (OPF) problem in a power system. The proposed algorithm is applied to determine the optimal settings of control variables of the OPF problem. The performance of the proposed algorithm was examined and tested on the standard IEEE 30bus test system with different objective functions and compared to other methods reported in the literature recently. Simulation results clearly obtained from the proposed (FA) approach indicated that (FA) provides an effective and robust high-quality solution for the OPF problem.
\end{abstract}

Keywords: Optimal Power Flow, Firefly Algorithm, Fuel Cost Optimization.

\section{INTRODUCTION}

The problem of optimal power flow (OPF) has received much attention and it is considered an important field of many utilities and it was marked as one of the most operational needs. The OPF problem solution aims to optimize a selected objective function via optimal adjustment of the power system control variables, while at the same time satisfying various equality and inequality constraints ${ }^{(\mathbf{1})}$.

The OPF problem is a large-scale highly constrained non-linear and non-convex optimization problem; it has taken decades to develop efficient algorithms for its solution. Many different mathematical techniques have been employed for its solution ${ }^{(2-3)}$. A wide variety of classical optimization techniques have been applied in solving the OPF problems considering a single objective function such as nonlinear programming ${ }^{(1-4)}$, quadratic programming ${ }^{(5-6)}$, linear programming ${ }^{(7-8)}$, Newton-based techniques ${ }^{(\mathbf{9 - 1 0})}$, sequential unconstrained minimization technique ${ }^{(\mathbf{1 1})}$, interior-point methods ${ }^{(\mathbf{1 2})}$. The non-linear programming optimization algorithm deals with problems involving nonlinear objective and constraint functions. Generally, non-linear programming-based procedures have many drawbacks such as insecure convergence properties and algorithmic complexity. The quadratic programming technique is a special form of non-linear programming whose objective function is quadratic with linear constraints. Quadratic programming based techniques have some disadvantages associated with the piecewise quadratic cost approximation. Newton-based techniques have a drawback of the convergence characteristics that are sensitive to the initial conditions and they may even fail to converge due to the inappropriate initial conditions. The sequential unconstrained minimization optimization techniques are known to exhibit numerical difficulties when the penalty factors become extremely large. Although the linear programming methods are fast and reliable, but they have some disadvantages associated with the piecewise linear cost approximation. The interior- point method converts the inequality constraints to equalities by the introduction of 


\section{K. Karboune et al.}

non-negative slack variables. This method has been reported as computationally efficient; however, if the step size is not chosen properly, the sub-linear problem may have a solution that is infeasible in the original non-linear domain. In additional, this method suffers from initial, termination, and optimality criteria and, in the most cases, is unable to solve nonlinear quadratic objective functions. For more discussions on these techniques, the reader can consult the comprehensive survey presented in Alsac and Stott ${ }^{(2)}$ and Momoh et al. (1999). In general, most of the classical optimization techniques mentioned apply sensitivity analysis and gradient-based optimization algorithms by linearizing the objective function and the system constraints around an operating point. Unfortunately, the OPF problem is a highly non-linear and a multimodal optimization problem, i.e. there exist more than one local optimum. Hence, local optimization techniques, which are well elaborated, are not suitable for such a problem.

Moreover, there is no criterion to decide whether a local solution is also the global solution. Therefore, conventional optimization methods that make use of derivatives and gradients are not able to identify the global optimum. Conversely, many mathematical assumptions such as convex, analytical, and differential objective functions have to be given to simplify the problem. However, the OPF problem is an optimization problem with in general non-convex, non-smooth, and non-differentiable objective functions. It becomes essential to develop optimization techniques that are efficient to overcome these drawbacks and handle such difficulties.

More recently, OPF has enjoyed renewed interest in a variety of formulations through the use of evolutionary optimization techniques to overcome the limitations of classical optimization techniques. A wide variety of advance optimization techniques have been applied in solving the OPF problems such as genetic algorithm $(\mathrm{GA})^{(\mathbf{1 4 - 1 5})}$, simulated annealing ${ }^{(\mathbf{1 6})}$, Tabu Search ${ }^{(\mathbf{1 7})}$, and particle swarm optimization (PSO) ${ }^{(\mathbf{1 8})}$. The results reported were promising and encouraging for further research in this direction. Unfortunately, recent research has identified some deficiencies in GA performance.

The degradation in efficiency is appeared in applications with highly epistatic objective functions, i.e. where the parameters being optimized are highly correlated. In addition, the premature convergence of GA degrades its performance and reduces its search capability. Recently, a new intelligent algorithm, called firefly algorithm (FA), has been proposed and introduced. The algorithm is inspired by biological and sociological motivations and can take care of optimality on rough, discontinuous and multi-modal surfaces. Ths algorithm mainly has three advantages: finding the true global minimum regardless of the initial parameter values, fast convergence, and has a few control parameters. Being simple, fast, easy to use, very easily adaptable for integer and discrete optimization, quite effective in non-linear constraint optimization including penalty functions and useful for optimizing multi-modal search spaces which represent the other important features of FA.

In the present study, a novel firefly approach was proposed to solve the OPF problem. The problem is formulated as an optimization problem. In this study, different objective functions were considered to minimize the fuel cost, to improve the voltage profile, and to enhance power system voltage stability. The proposed approach has been examined and tested on the standard IEEE30-bus test system.

The potential and effectiveness of the proposed approach are demonstrated. Additionally, the results were compared to those reported in the literatures. The OPF problem solution aims to optimize a selected objective function via optimal adjustment of the power system control variables, while at the same time satisfying various equality and inequality constraints. Mathematically, the OPF problem can be formulated as follows ${ }^{(\mathbf{1})}$ : 


\section{Optimal Power Flow Using firefly-algorithm}

$\operatorname{Min} f(x, u)$

Subject to: $g(x, u)=0$

$h(x, u) \leq 0$

where $f$ is objective function to be minimized.

$x$ is the vector of dependent variables (state vector) consisting of:

1. Active generating power at slack bus $P_{\mathrm{G} 1}$.

2. Load-bus voltage $V_{\mathrm{L}}$.

3. Generator reactive power outputs $Q_{G}$.

4. Transmission line loading (or line flow) $S_{l}$.

Hence, $x$ can be expressed as:

$x^{T}=\left[P_{G 1}, V_{L 1} \ldots V_{L N L}, Q_{G 1} \ldots Q_{G N G}, S_{l 1} \ldots S_{l n}\right]$

where, NL, NG, and nl are the number of load buses, number of generators, and number of transmission lines, respectively.

$u$ is the vector of independent variables (control variables)consisting of:

1. Generator voltage $V_{G}$ at PV buses.

2. Generator real power output $P_{G}$ at PV buses except at the slack bus $P_{\mathrm{Gl}}$.

3. Transformer tap setting $T$.

4. Shunt VAR compensation (or reactive power of switchable VAR sources) $Q_{c}$.

Hence, $u$ can be expressed as:

$u^{T}=\left[P_{G 2} \ldots P_{G \mathrm{NG}}, V_{G 1} \ldots V_{G \mathrm{NG}}, Q_{c 1} \ldots Q_{c \mathrm{Nc}}, T_{1} \ldots T_{\mathrm{NT}}\right]$

where, NT and NC are the number of the regulating transformer and VAR compensators, respectively. $g$ is the equality constraints represent typical load flow equations.

$$
\begin{aligned}
& P_{G i}-P_{D i}-V_{i} \sum_{i=1}^{N} V_{j}\left(G_{i j} \cos \theta_{i j}+B_{i j} \sin \theta_{i j}\right)=0 . \\
& Q_{G i}-Q_{D i}-V_{i} \sum_{i=1}^{N} V_{j}\left(G_{i j} \cos \theta_{i j}-B_{i j} \sin \theta_{i j}\right)=0
\end{aligned}
$$

where, $i=1, \ldots, \mathrm{N}$;

$\mathrm{N}$ is the number of buses;

$P_{G}$ is the active power generated,

$Q_{G}$ is the reactive power generated,

$P_{\mathrm{D}}$ is the load active power;

$Q_{\mathrm{D}}$ is the load reactive power,

$G_{i j}$ and $B_{i j}$ are the transfer conductance and susceptance between bus $i$ and bus $j$, respectively. $h$ is the system operating constraints that includes:

1. Generator constraints: generator voltages, real power outputs, and reactive power outputs are restricted by their lower and upper limits as follows:

$$
\begin{aligned}
& V_{G i}^{\min } \leq V_{G i} \leq V_{G i}^{\max }, \quad i=1, \ldots, \mathrm{NG} \\
& P_{G i}^{\min } \leq P_{G i} \leq P_{G i}^{\max }, \quad i=1, \ldots, \mathrm{NG} \\
& Q_{G i}^{\min } \leq Q_{G i} \leq Q_{G i}^{\max }, \quad i=1, \ldots, \mathrm{NG}
\end{aligned}
$$

2. Transformer constraints: transformer tap settings are bounded as follows:

$T_{G i}^{\min } \leq T_{i} \leq T_{G i}^{\max }, \quad i=1, \ldots, \mathrm{NT}$

3. Shunt VAR constraints: shunt VAR compensations are restricted by their limits as follows:

$Q_{c i}^{\min } \leq Q_{c i} \leq Q_{c i}^{\max }, \quad i=1, \ldots, \mathrm{NC}$ 


\section{K. Karboune et al.}

4. Security constraints: these include the constraints of voltages at load buses and transmission line loadings as follows:

$$
\begin{aligned}
& V T_{L i}^{\min } \leq V_{L i} \leq V_{L i}^{\max }, \quad i=1, \ldots, \mathrm{NL} \\
& S_{L i} \leq S_{G i}^{\min }, \quad i=1, \ldots, \mathrm{nl} \ldots \ldots \ldots \ldots \ldots \ldots \ldots \ldots \ldots
\end{aligned}
$$

\section{Firefly Algorithm (FA)}

\section{RESULTS AND DISCUSSION}

The firefly algorithm (FA) is another swarm intelligence algorithm, developed by Xin-She Yang ${ }^{(\mathbf{1 9 )}}$,that evolves fast for almost area of optimization and engineering problems. Stand alone firefly algorithm already has managed to solve problems. For problems that have multi dimensional and nonlinear problem, some modification or even hybridization with the other metaheuristic is advisable. This modification and hybridization will help for the computational constrain and it will become more flexible and more efficient ${ }^{\mathbf{2 0 , 2 1})}$. The Firefly Algorithm (FA) is based on the communication behaviour of tropical fireflies and the idealized behaviour of the flashing patterns. FA uses the following three idealized rules ${ }^{(22-23)}$ to build the mathematical model of the algorithm:

- All fireflies are unisex so that one firefly will be attracted to other fireflies regardless of their sex;

- Attractiveness is proportional to their brightness. Thus for any two flashing fireflies, the less bright one will move towards the brighter one. The attractiveness is proportional to the brightness and they both decrease as their distance increases;

- The brightness of a firefly is affected or determined by the landscape of the objective function. (Thus, for a maximization problem, the brightness can simply be proportional to the value of the objective function.)

In the standard firefly algorithm, there are two important points. One is the formulation of the light intensity and another is the change of the attractiveness. Firstly, we can always assume that the brightness of the firefly can be determined by the encoded objective function landscape.

Secondly, we should define the variation of light intensity and formulate the change of the attractiveness. As we know that in nature the light intensity decreases with the distance from its source and the media will absorb the light, so in our simulation we suppose the light Intensity $I$ varies with the distance $r$ and light absorption parameter $\gamma$ exponentially and monotonically ${ }^{(24)}$. That is

$$
I=I_{0} e^{-\gamma r^{2}}
$$

where $I_{0}$ is the original light intensity at the source (i.e., at the distance $r=0$ ) and $\gamma$ is the light absorption coefficient. From the idealized rules we known that in our simulation we suppose the attractiveness of firefly is proportional to the light intensity $I$. So we can define the firefly's light attractive coefficient $\beta$ in the similar way as the light intensity coefficient $I$. That is

$$
\beta=\beta I_{0} e^{-\gamma r^{2}}
$$

where $\beta_{0}$ is the original light attractiveness at $r=0$.

The Cartesian distance is used to calculate the distance between any two fireflies $i$ and $j$ at $x_{i}$ and $x_{j}$

$$
r_{i j}=\left\|x_{i}-x_{j}\right\|_{2}=\sqrt{\sum_{k-1}^{d}\left(x_{i, j}-x_{j, k}\right)^{2}}
$$

where $d$ is the number of dimensions. The amount of movement of firefly $i$ to another more attractive (brighter) firefly $j$ is determined by

$$
x_{i}=x_{i}+\beta_{0} e^{-\gamma r^{2}}\left(x_{j}-x_{i}\right)+\alpha \varepsilon_{i}
$$




\section{Optimal Power Flow Using firefly-algorithm}

where the first terms the current location of firefly $i$, the second terms due to the attraction, while the third termis randomization with the vector of random variables $\varepsilon_{i}$ being drawn from different distributions such as the Uniform distribution, Gaussian distribution and Lévy flight.

In the third term, $\alpha$ is a scaling parameter that controls the step size and it should be linked with the interests of the problems.

According to the above idealization and approximations rules, the pseudo-code of standard firefly algorithm can be summarized in Algorithm 1.

Objective function $\mathrm{f}(\mathrm{x}), \mathrm{x}=\left(\mathrm{x}_{1},{ }_{-},-, \mathrm{x}_{\mathrm{D}}\right)^{\mathrm{T}}$

Initialize a population of fireflies $x_{i}\left(i=1,2,{ }_{-}-n\right)$

Calculate the light intensity Ii at xi by $\mathrm{f}\left(\mathrm{x}_{\mathrm{i}}\right)$

Define light absorption coefficient $\gamma$

While ( $\mathrm{t}<$ Max Generation)

for $\mathrm{i}=1: \mathrm{n}$ all $\mathrm{n}$ fireflies

for $\mathrm{j}=1: \mathrm{n}$ all $\mathrm{n}$ fireflies

Calculate the distance $\mathrm{r}$ between $\mathrm{xi}$ and $\mathrm{x}_{\mathrm{j}}$ using Cartesian distance equation if $\left(\mathrm{I}_{\mathrm{j}}>\mathrm{I}_{\mathrm{i}}\right)$

Attractiveness varies with distance $r$ via $\beta_{0} e^{-\gamma r^{2}}$

Move firefly $\mathrm{i}$ towards $\mathrm{j}$ in all $\mathrm{d}$ dimensions end if Evaluate new solutions and update light intensity end for $\mathrm{j}$ end for $\mathrm{i}$

Rank the fireflies and find the current best end while Algorithm Pseudo-code for the FA.

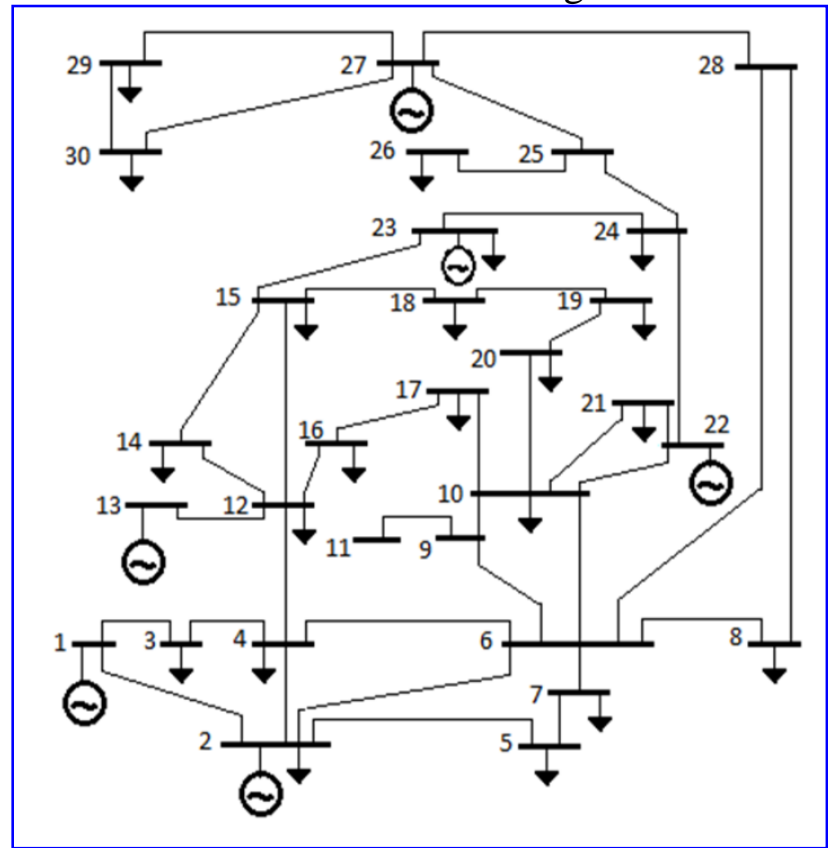

Fig. 1. Single-line diagram of IEEE 30-bus test system.

The IEEE 30-bus system has been investigated here as shown in Fig.1 to present the efficiency of the proposed algorithms (FA) and Particle Swarm Optimization (PSO). The line data and the bus data are taken from Valenzuela and Smith ${ }^{(\mathbf{2 9})}$. The system real and reactive power demands are respectively 2.834 p.u and 1.262 p.u at 100 MVA base. One case is selected to examine the basic objective function. The FA and PSO algorithms have been implemented to solve the OPF problem for one case illustrated as objective function. The population and generation number for each algorithm are 15 and 50 respectively. The bounds of control variables are given in Valenzuela and Smith ${ }^{(\mathbf{2 9})}$. The simulation execution has been carried out under Matlab software. 


\section{K. Karboune et al.}

Table 1. (Comparison of cost for PSO method)

\begin{tabular}{|c|c|c|}
\hline \multicolumn{3}{|c|}{ Method } \\
\hline Unit & FA & PSO \\
\hline P1 & 176.784 & 154,647 \\
\hline P2 & 48.841 & 48,826 \\
\hline P3 & 21.483 & 21,382 \\
\hline P4 & 21.618 & 21,740 \\
\hline P5 & 12.053 & 11,722 \\
\hline P6 & 12.0 & 33,194 \\
\hline Fuel cost (\$/hr) & $\mathbf{8 0 1 . 8 4 3 7}$ & 801,855 \\
\hline
\end{tabular}

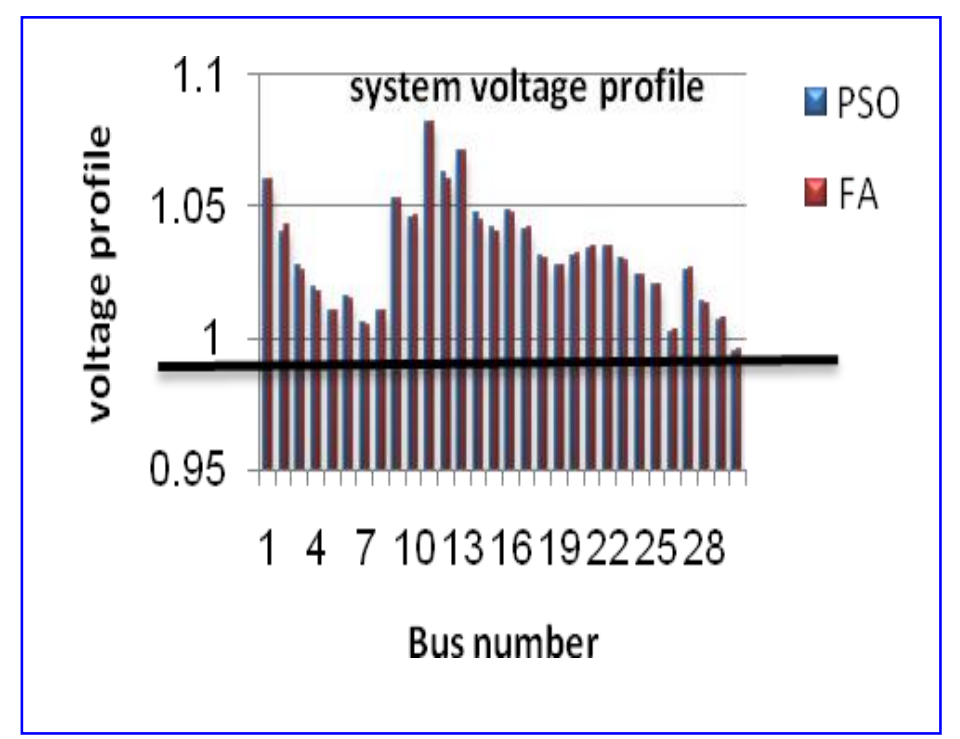

Fig. 2. System voltage profile

The control variable parameters achieved by the FA and the values the objective function with different algorithms are exposed in the table 1. In addition, a comparative study with another algorithm has been done in order to solve the single objective OPF problems. From Tables $(1 \& 2)$ it can be observed that, the FA method performed and supplied remarkable results compared with PSO method and other algorithms reported in the literature. Also, the voltage profile enhancement at all buses is clearly attained in case of FA algorithm and illustrated in Figure (2).

Table 2. Comparison of cost for different methods.

\begin{tabular}{|l|l|l|}
\hline Method & Author & Cost $(\$ / \mathrm{hr})$ \\
\hline Gradient-based Approach & 25 & 804.853 \\
\hline Evolutionary Programming & 27 & 802.62 \\
\hline $\begin{array}{l}\text { Nonlinear Programming } \\
\text { solution }\end{array}$ & 28 & 802.40 \\
\hline Tabu Search Algorithm & 26 & 802.29 \\
\hline Firefly Algorithm & Present & $\mathbf{8 0 1 . 8 4 3 7}$ \\
\hline
\end{tabular}




\section{Optimal Power Flow Using firefly-algorithm}

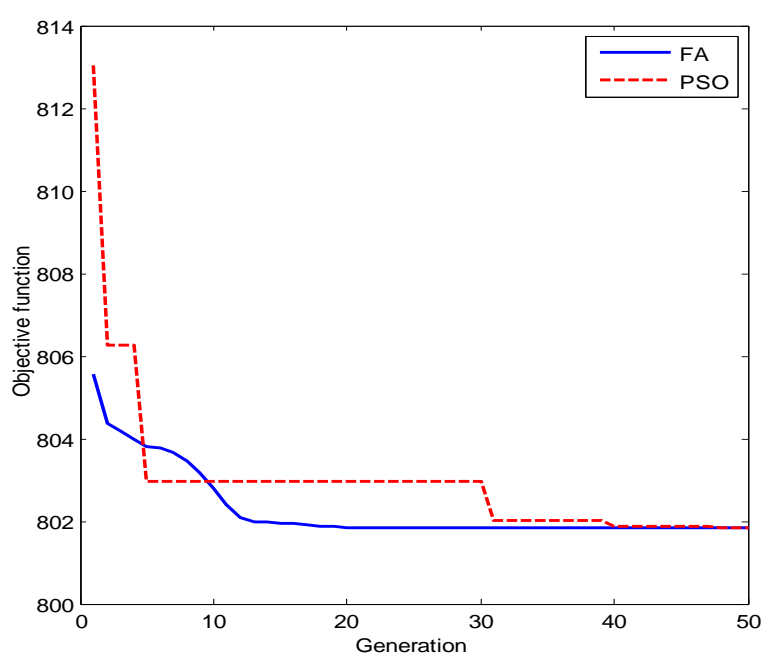

Fig. 3. Objective function OPF problems

The convergence curves of the total cost for the proposed algorithms are illustrated in Figure (3). It is quite noticeable that the FA has the best convergence characteristics with best value of objective function. FA converges better than PSO towards a final exact solution.

\section{Conclusion}

In this study, a novel algorithm called FA has been shown and employed to solve the OPF problem. The fuel cost has been considered the main objective function. FA was applied to obtain minimum value of the objective function. Real power of the generators was chosen as control variables during this study. The IEEE 30-bus system has been presented here to examine the performance of the proposed algorithms. The simulation results obviously proved that the proposed FA algorithm achieves best value of the objective function as compared with PSO algorithm.

\section{REFERENCES}

1. Abou El Ela, A.A.; Abido, M.A. and Spea, S.R. (2009). Optimal power flow using differential evolution algorithm. Electr.Eng., 91: 69-78. https://doi.org/10.1007/s00202009-0116-Z

2. Alsac, O. and Stott, B. (1974). Optimal Load Flow with Steady-State Security. In: IEEE Transactions on Power Apparatus and Systems, vol. PAS-93, no. 3, pp. 745-751.

3. Momoh, J. A.; El-Hawary, M. E. and Adapa, R. (1999). A review of selected optimal power flow literature to 1993. II. Newton, linear programming and interior point methods. In: IEEE Transactions on Power Systems, 14(1):105-111.

4. Habibollahzadeh, H.; Luo, G.X. and Semlyen, A. (1989). Hydrothermal optimal power flow based on a combined linear and non-linear programming methodology. IEEE Trans Power Apparatus Syst PWRS-4(2):530-537

5. Burchett, R.C.; Happ, H.H. and Vierath, D.R. (1984). Quadratically convergent optimal power flow. IEEE Trans Power Apparatus Syst PAS- 103:3267-3276

6. Reid, G.F. and Hasdorf, L. (1973). Economic dispatch using quadratic programming. IEEE Trans Power Apparatus Syst PAS-92: 2015-2023

7. Stadlin, W. and Fletcher, D. (1982). Voltage verus reactive current model for dispatch and control. IEEE Trans Power Apparatus Syst PAS- 101(10):3751-3758. 


\section{K. Karboune et al.}

8. Abou El Ela, A.A.; Abido, M.A. and Spea, S.R. (2009). Optimal power flow using differential evolution algorithm. Electr.Eng., 91:69-78.https://doi.org/10.1007/s00202009-0116-Z

9. Sun, D.I.; Ashley, B.; Brewer, B.; Hughes, A. and Tinney, W.F. (1984). Optimal Power Flow by Newton Approach. IEEE Transaction on Power Apparatus and Systems, PAS103,2864-2880.

10. Santos, A. (1995). Optimal power flow solution by Newton's method applied to AN augmented lagrangian function. IEE Proc Gener Transm. Distrib., 142(1):33-36

11. Rahli, M. (1999). Optimal power flow using sequential unconstrained minimization technique (SUMT) method under power transmission losses minimization. Electr. Power Syst. Res., 52:61-64

12. Momoh, J.A. (1999). Improved interior point method for OPF problems.IEEE Trans Power Apparatus Syst., 14(3):1114-1120

13. Momoh, J.A.; El-Hawary, M.E. and Adapa, R. (1999). A review of selected optimal power flow literature to 1993. Part I: Non-linear and quadratic programming approaches. IEEE Trans Power Syst., 14(1): 96-104

14. Lai, L.L.; MA. J.T.; Yokohoma, R. and Zhao, M. (1997). Improved genetic algorithm for optimal power flowunder both normal and contingent operation states. Electr Power Energy Syst., 19:287-291

15. Osman, M.S. and Abo-Sinna, M.A (2003). A solution to the optimal power flow using genetic algorithm. Elsevier, Amsterdam

16. Miranda, V.; Srinivasan, D. and Proenca, L.M. (1999). Evolutionary computation in power systems. Electr Power Energy Syst., 20:89-98

17. Abido, M.A. (2002). Optimal power flow using tabu search algorithm. Electr. Power Compon. Syst., 30(5):469-483

18. Abido, M.A. (2002). Optimal power flow using particle swarm optimization. Int. J. Electr. Power Energy Syst., 24(7):563-571

19. Yang, X.S. (2008). Nature-Inspired Metaheuristic Algorithms, Luniver Press.

20. Nadhirah, A.; Mohd, Ah. O.; Mohd, N.H. and Mohamad, H.M. (2014). A review of firefly algorithm. ARPN J. Engineering and Appl. Sci., 9(10).

21. Zhang, L., Liu, L.; Yang, X-S and Dai, Y. (2016). A Novel Hybrid Firefly Algorithm for Global Optimization. PLoS ONE 11(9): doi:10.1371/journal.pone.0163230

22. Yang X.S. (2009). Firefly algorithms for multimodal optimization. Stochastic algorithms: foundations and applications. Springer Berlin Heidelberg. 2009, pp. 169-178

23. Marichelvam, M.K. and Geetha, M.A. (2016). Hybrid discrete firefly algorithm to solve flow shop scheduling problems to minimize total flow time. International Journal of BioInspired Computation, 8(5):318-325. DOI:10.1504/IJBIC.2016.079572

24. Yang X.S. (2910). Firefly algorithm, Lévy flights and global optimization. Research and development in intelligent systems XXVI. Springer London. pp. 209-218.

25. Lee, K.; Park, Y. and Ortiz, J. (1985). A united approach to optimal real and reactive power dispatch. IEEE Trans Power Apparatus Syst., 104(5):1147-115333.

26. ABIDO, M.A. (2002). Optimal Power Flow Using Tabu Search Algorith, Electric Power Components and Systems, 30:469-483.

27. Yuryevich, J. and Wong, K.P. (1999). Evolutionary Programming Based Optimal Power Flow Algorithm. IEEE Trans. on Power Systems, 14(4):1245-1250.

28. Alsac, O. and Stott, B. (1974). Optimal Load Flow with Steady State Security. IEEE Trans. on Power Apparatus and Systems, Vol. PAS-93, pp. 745-751. 


\section{Optimal Power Flow Using firefly-algorithm}

29. Valenzuela, J. and Smith, A. (2002). A seeded memetic algorithm for large unit commitment problems. J. Heuristics, 8(2):173-195. 